\section{Phosphornanostränge - Zwei neue Modifikationen des Phosphors}

\author{
Michael F. Bräu ${ }^{a}$, Arno Pfitzner ${ }^{a}$, *, Josef Zweck ${ }^{b}$, \\ Gunther Brunklaus ${ }^{\mathrm{c}}$, Hellmut Eckert ${ }^{\mathrm{c}}$ \\ a Institut für Anorganische Chemie, Universität Regensburg, \\ D-93040 Regensburg \\ b Institut für Experimentelle und Angewandte Physik, Universität \\ Regensburg, D-93040 Regensburg \\ c Institut für Physikalische Chemie, Westfälische Wilhelms- \\ Universität, D-48149 Münster
}

Keywords: Phosphorus; Nanorod; Polymer; Copper iodide

Bisher wurden vier Modifikationen des Phosphors strukturell aufgeklärt [1-3]. Wir konnten zwei neue Modifikationen aus gut charakterisierten Festkörperverbindungen erhalten. Diese sind aus Polymersträngen ähnlich denen im violetten Phosphor aufgebaut. Das erste Polymer wurde aus $(\mathrm{CuI})_{8} \mathrm{P}_{12}$ isoliert. Wie in $\mathrm{Abb} .1$ gezeigt, besteht es aus $\mathrm{P}_{8}$-Käfigen, die über quadratische $\mathrm{P}_{4}$-Einheiten verbunden sind. Das zweite Polymer wurde aus $(\mathrm{CuI})_{3} \mathrm{P}_{12}$ extrahiert. Es setzt sich aus $\mathrm{P}_{10}$-Käfigen zusammen, die über $\mathrm{P}_{2}$-Hanteln verbunden sind, wie in Abbildung 2 zu sehen ist.

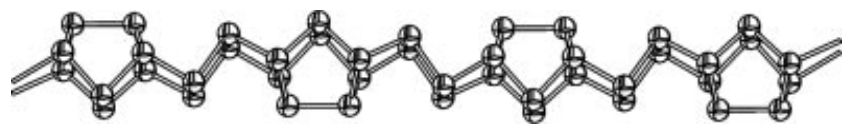

Abb. 1 Ein $\left.{ }_{\infty}^{1}\right] \mathrm{P} 4(4)[\mathrm{P} 8]$ Strang aus $(\mathrm{CuI})_{8} \mathrm{P}_{12}$.

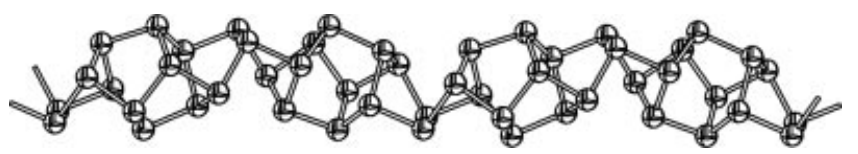

Abb. 2 Ein $\left.{ }_{\infty}^{1}\right] \mathrm{P} 2[\mathrm{P} 10]-$ Strang aus $(\mathrm{CuI})_{3} \mathrm{P}_{12}$.

In den Eduktverbindungen liegen die Polymerstränge in einer $\mathrm{Ku}-$ pferiodid-Matrix eingebettet vor [4, 5]. Aus dieser Matrix werden sie durch Extraktion des Kupferiodids mit wässriger Kaliumcyanidlösung isoliert. Dabei fallen die Polymerstränge als feines, rotbraunes, faseriges Pulver an, welches bei Raumtemperatur an Luft stabil ist. Sie ordnen sich in einer dichten Stabpackung an und bilden so feine Fasern. Die beiden neuen Phosphormodifikationen wurden mit Röntgenpulverdiffraktion, Festkörper ${ }^{31} \mathrm{P}-\mathrm{MAS}-\mathrm{NMR}$ und HR-TEM charakterisiert. Der Durchmesser der Stränge beträgt für das Polymer aus $(\mathrm{CuI})_{8} \mathrm{P}_{12} 5.0 \AA$, für das aus $(\mathrm{CuI})_{3} \mathrm{P}_{12}$ $5.4 \AA$.

[1] A. Simon, H. Borrmann, Phosphorus, Sulphur Silicon Relat. Elem. 1987, 30, 507.

[2] H. Thurn, H. Krebs, Angew. Chem. 1966, 78, 1101; Angew. Chem. Int. Ed. 1966, 5, 1047.

[3] D. E. C. Corbridge, Phosphorus 2000, Elsevier, Amsterdam, 2000.

[4] M. Möller, W. Jeitschko, Solid State Chem. 1986, 65, 178.

[5] A. Pfitzner, E. Freudenthaler, Angew. Chem. 1995, 107, 1784; Angew. Chem. Int. Ed. 1995, 34, 1647.

\section{Zur Anionenverteilung in $\mathrm{Zr}_{2} \mathrm{ON}_{2}$}

\section{Thomas Bredow ${ }^{\mathrm{a}}$, Martin Lerch}

a Theoretische Chemie, Universität Hannover, Am Kleinen Felde 30, D-30167 Hannover

b Institut für Chemie, Technische Universität Berlin, Straße des 17. Juni 135, D-10623 Berlin

Keywords: Anion distribution, oxynitrides, Theoretical study, Electronic structure

$\mathrm{Zr}_{2} \mathrm{ON}_{2}$ kristallisiert in der Bixbyitstruktur [1-3], die als eine Anioniendefizit-Variante des Fluorit-Typs mit geordneten Anionenleerstellen betrachtet werden kann (1/4 der Anionenplätze sind unbesetzt). Die Anionenverteilung in $\mathrm{Zr}_{2} \mathrm{ON}_{2}$ ist in der Literatur umstritten. Füglein et al. [2] berichten über das Vorliegen einer geordneten Verteilung von Stickstoff und Sauerstoff im Anionengitter (Raumgruppe $I b c a$ ). Clarke et al. [3] geben einer statistischen Verteilung der Anionen (Raumgruppe $I a \overline{3}$ ) den Vorzug.

Die hier präsentierte theoretische Studie untersucht die Stabilität von verschiedenen Anordnungen der Anionen mittels periodischer quantenchemischer Rechnungen auf DFT-Niveau. Dies wird ergänzt durch rein elektrostatische Kalkulationen und Rechnungen zur elektronischen Struktur.

In Kombination mit einfachen thermodynamischen Betrachtungen ergibt sich die energetisch günstigste Situation beim Vorliegen einer statistischen Verteilung der Anionen. Die relative Stabilität der Strukturen lässt sich mit der mittleren Koordination der Anionen korrelieren (siehe Abbildung). Das energetische Minimum befindet sich in der Nähe der mittleren Koordinationszahlen für eine statistische Anionenverteilung.

Die berechnete elektronische Struktur mit einer Bandlücke von 2.5 $\mathrm{eV}$ stimmt gut mit den experimentellen Beobachtungen überein. Die Studien werden von der DFG im Rahmen des SPP 1136 gefördert.

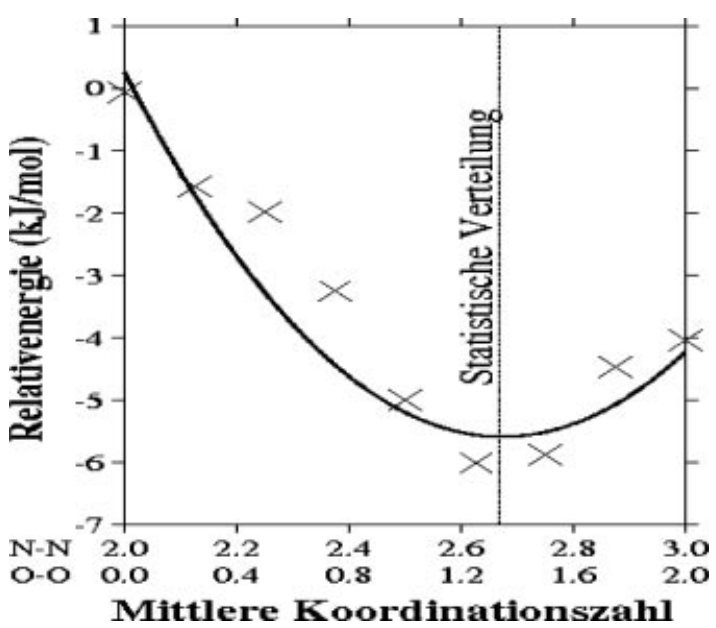

[1] J. C. Gilles, Bull. Soc. Chim. Fr. 1962, 22, 2118.

[2] E. Füglein, R. Hock, M. Lerch, Z. Anorg. Allg. Chem. 1997, 623, 304.

[3] S. J. Clarke, C. W. Michie, M. J. Rosseinsky, Chem. Mater. 1999, $146,399$. 